\title{
HYALINISING TRABECULAR TUMOUR OF THE THYROID GLAND- A DIAGNOSTIC CHALLENGE
}

\author{
Hema Kini' ${ }^{1}$, Saraswathy Sreeram², Vijendra Shenoy33, Nirupama Murali ${ }^{4}$
}

1 Professor \& HOD, Department of Pathology, Manipal University, Kasturba Medical College, Mangalore. ${ }^{2}$ Assistant Professor, Department of Pathology, Manipal University, Kasturba Medical College, Mangalore.

${ }^{3}$ Professor \& HOD, Department of Otorhinolaryngology, Manipal University, Kasturba Medical College, Mangalore. ${ }^{4}$ Associate Professor, Department of Pathology, Manipal University, Kasturba Medical College, Mangalore.

HOW TO CITE THIS ARTICLE: Kini H, Sreeram S, Shenoy V, et al. Hyalinising trabecular tumour of the thyroid gland- A diagnostic challenge. J. Evolution Med. Dent. Sci. 2017;6(66):4790-4792, DOI: 10.14260/Jemds/2017/1038

\section{PRESENTATION OF CASE}

A 42-year-old housewife presented to the Otorhinolaryngology Outpatient Department with a painless swelling of left side of the neck, insidious in onset, gradually increasing in size since 1 year. On local examination, the swelling measured $4 \mathrm{~cm} \times 3 \mathrm{~cm}$ and was nodular, firm, mobile, nontender, and moving with deglutition. Cervical lymphadenopathy was absent. Skull and spine were normal. Thyroid function tests revealed that she was euthyroid. Ultrasonography of the neck showed a well-defined nodule on the left thyroid lobe with homogenous echotexture. Fine needle aspiration cytology (FNAC) was performed elsewhere and a diagnosis of papillary carcinoma was given. She came to our department to undergo total thyroidectomy with lymph node dissection, which is the standard surgical management for papillary carcinoma. Her internal medicine practitioner gave reference to our tertiary care centre for the same.

\section{DIFFERENTIAL DIAGNOSES}

The most common differential diagnoses for unilateral enlargement of thyroid gland at this age include colloid cyst, hyperplastic nodule, adenoma and carcinoma. Since our lady already had undergone needle aspiration cytology and had a report of papillary carcinoma with her, we went ahead with the routine anaesthesia checkup and pre-operative investigations for assessing surgical fitness. Since everything was normal, we went ahead with the surgical procedure. Total thyroidectomy was performed. There were no enlarged cervical lymph nodes intra-operatively. The post-surgery period was uneventful and the patient was lost to follow-up.

\section{PATHOLOGICAL DISCUSSION}

We received the surgical specimen in the Pathology Department. Cut surface of the left lobe of thyroid showed a well-circumscribed, firm, solid grey-white nodule completely replacing the lobe (Figure 1a). The right lobe and isthmus showed normal thyroid parenchyma filled with colloid. The most common differential diagnoses of encapsulated thyroid lesions are macrofollicular adenoma, adenomatoid nodule, follicular adenoma, follicular variant of papillary carcinoma and an encapsulated follicular carcinoma.

Financial or Other, Competing Interest: None.

Submission 13-07-2017, Peer Review 06-08-2017,

Acceptance 12-08-2017, Published 17-08-2017.

Corresponding Author:

Saraswathy Sreeram,

Department of Pathology,

Lighthouse Hill Road,

Hampankatta,

Mangalore- 575001, Karnataka.

E-mail: swameeram@gmail.com

DOI: $10.14260 /$ jemds $/ 2017 / 1038$

\section{(c) $(1)(5)$}

Histological sections from the left lobe showed a tumour covered by a thin capsule. The cells were arranged in trabecular pattern separated by delicate fibrovascular septae (Figure 1b). The cells were oriented perpendicular to the long axis of the trabeculae. The cytoplasm was pale to eosinophilic. Nuclei were enlarged, round to oval, with granular chromatin, inconspicuous nucleoli, frequent nuclear grooves and occasional intranuclear inclusions along with perinuclear halos (Figure 1c). Intertrabecular and intratrabecular hyaline was present. Mitotic figures were rare. There was no evidence of necrosis, capsular invasion or vascular invasion. Congo red staining was negative for amyloid (Figure 1d). The right lobe and isthmus showed unremarkable thyroid parenchyma made up of follicles filled with colloid. Immunohistochemistry was done in our case and showed a cytoplasmic granular positivity with Ki-67, however, there was no membrane positivity (Figure 1e).

The differentials for tumours with trabecular growth pattern include hyalinising trabecular tumour (HTT), medullary carcinoma, follicular (Hurthle cell) adenoma or carcinoma, papillary carcinoma and poorly differentiated carcinoma.

Since careful sampling and multiple sections from the specimen did not reveal any focus of papillary carcinoma, we retrieved the cytology smears from the laboratory where the aspiration was performed. The smears showed cohesive aggregates of cells arranged in syncytial clusters and singly (Figure 1f). These cells were focally oriented around small irregular fragments of hyaline material (Figure 1g). Cells had ample cytoplasm, overlapping, enlarged nuclei with irregular membranes and intranuclear grooves (Figure $1 \mathrm{~h}$ ). Background was haemorrhagic and colloid was scant. Psammoma bodies and cytoplasmic yellow bodies were absent.

HTT is frequently (80\%) misdiagnosed as papillary carcinoma on cytology.[1-3] Miller and co-workers advised following a three criteria rule to avoid false-positive diagnosis of papillary carcinoma on cytology. These include the presence of intranuclear inclusions, metaplastic cytoplasm and avascular papillary structures. The presence of at least two of the three features is necessary to diagnose a papillary carcinoma on cytology.[4] Presence of intratrabecular hyaline favours a diagnosis of HTT.[3] Our case was diagnosed as papillary carcinoma on cytology based on the nuclear features alone, despite a lack of papillary architecture or a sheet-like pattern. Chewing-gum colloid seen in papillary carcinoma and the hyalinised stroma seen in HTT are indistinguishable in cytology and hence misinterpreted to be thick colloid. Chewing-gum colloid appears as strands or chunks of dense colloid.

HTT is also mistaken for a medullary carcinoma on cytology owing to its occasional spindle cell morphology, 
trabecular and dispersed patterns, and hyaline confused with amyloid. Hyaline appears as acellular lumpy amorphous material. Amyloid on aspiration is fluffy or fibrillar, acellular and stains positive with Congo red. Cytoplasmic red granules are also a characteristic feature of medullary carcinoma.[5]

The distinguishing features of HTT in cytology are the radial arrangement of cohesive tumour cells surrounding the hyaline material, whirling parallel arrays, vague, curved nuclear palisading, spindled or elongated cells, ill-defined cell borders, faintly stained, abundant, filamentous cytoplasm, frequent intranuclear grooves, pseudoinclusions, perinuclear halo, haemorrhagic background, lack of papillary architecture and sheet-like arrangement.[2]

In correlation to all the features described above, we gave the final impression as HTT.

HTT of the thyroid gland is an unusual tumour first described by Carney et al in 1987 as an adenoma. The World Health Organization (WHO) classification currently classifies it as an independent entity and has replaced the term "adenoma" with "tumour". It is defined as "a rare tumour of follicular cell origin with a trabecular pattern of growth and marked intra-trabecular hyalinisation".[6] Diagnosis of this tumour based on cytology alone is difficult owing to its similarity with papillary and medullary carcinomas.

HTT is a rare tumour derived from follicular epithelium that has been reported more in females between 30-50 years of age. These are associated with lymphocytic thyroiditis. It is quite a controversial entity especially with respect to its clinical behaviour. Almost all the classical cases have not shown capsular or vascular invasion or distant metastasis. Rare cases of hyalinising trabecular carcinoma have been reported with recurrence and metastases to lung and lymph nodes.[6]

HTTs are generally small, circumscribed lesions $(<2 \mathrm{~cm})$ with solid yellow tan appearance of cut surface. Its main histological features consist of a trabecular pattern, with variable deposition of PAS-positive hyaline material in the cytoplasm (intermediate filaments) as well as in the extracellular spaces (collagen fibres and basement membrane material).[6] The tumour cells are medium to large, polygonal to fusiform with eosinophilic granular cytoplasm, nuclei with grooves and inclusions. A characteristic feature seen in this tumour is presence of cytoplasmic pale yellow bodies that are perinuclear, 2-5 $\mu \mathrm{m}, \quad$ spherical and refractile. Ultrastructurally, they conform to giant lysosomes.[7] Though they found the feature in Hurthle cell tumours (83\%), papillary carcinoma (62\%) and follicular carcinoma (22\%), Rothenberg et al reported it to be a diagnostically useful feature of HTTs (100\%).

Its relationship with papillary carcinoma has been debated. It was considered to be an encapsulated variant of papillary carcinoma due to similar nuclear features, presence of psammoma bodies, immunohistochemistry profile and RET/PTC translocation. However, BRAF mutations were absent in all cases of HTT.[8]

Immunohistochemistry shows HTT to be positive for thyroglobulin, TTF-1 and galectin-3 (variable). Cytokeratin19, high molecular weight cytokeratin, HBME-1, neuroendocrine markers and calcitonin are usually negative in HTT. ${ }^{[2]}$ The strong granular cytoplasmic and membrane MIB-1 (Ki-67) expression of HTT (71\%) has been reported to be helpful in distinguishing this tumour from papillary carcinoma. ${ }^{[9]}$ However, a study by Boerner and co-workers concluded otherwise.[10] (Figure 1f).

Another rare differential of HTT is primary thyroid paraganglioma. Both can show intranuclear cytoplasmic inclusions. A fine red cytoplasmic granulation is often seen in paragangliomas while intratrabecular hyaline and cytoplasmic yellow bodies are characteristic of HTT. They can also be differentiated based on positive immunoreactivity of paragangliomas with neuroendocrine markers and negativity with thyroglobulin. [3]

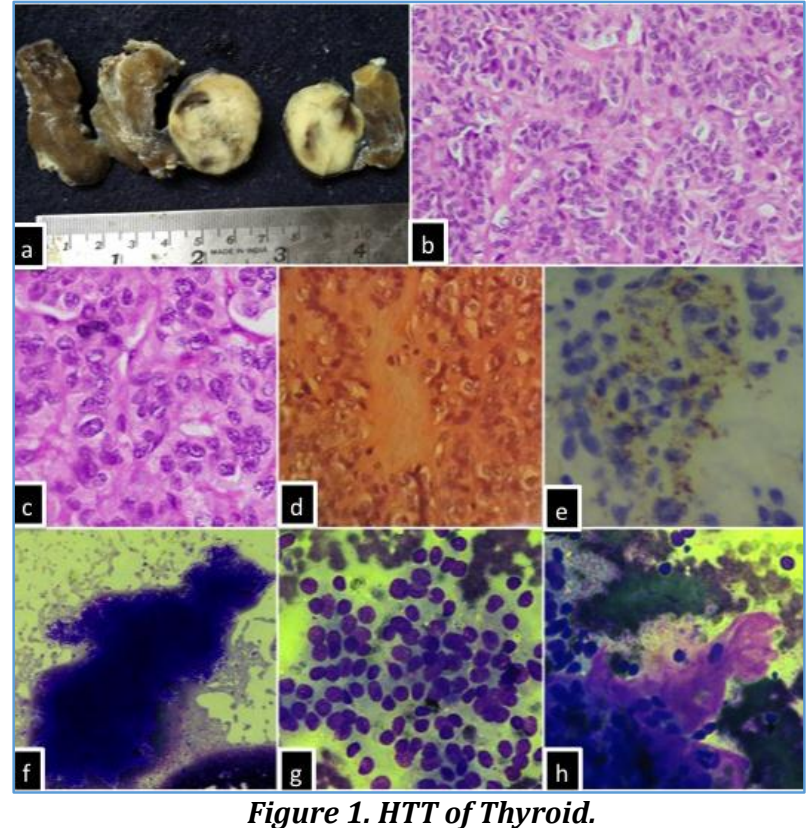

a. Gross: Well-circumscribed Tumour in the Right Lobe of Thyroid

b. Tumour in Trabecular Pattern with Intratrabecular Hyalinisation, Haematoxylin \& Eosin (H\&E), 10x

c. Tumour Cells with Clear Cytoplasm, Nuclear Grooves, $H \& E, 40 x$

d. Congo Red Stain Negative for Amyloid, 40x

e. Strong Cytoplasmic Granular Positivity with Ki-67, IHC, 40x

f. Syncytial Arrangement of Cells on Cytology, May Grunwald Giemsa (MGG), 4x

g. Occasional Nuclear Grooves, MGG, 20x

h. Eosinophilic Hyaline-like Material, MGG, 10x

\section{DISCUSSION OF MANAGEMENT}

HTT is a challenging diagnosis on cytology. It is important to distinguish it from papillary carcinoma because of the differing management strategies. Lobectomy with annual follow-up for any recurrence is considered sufficient for HTT whereas total thyroidectomy followed by radioactive iodine therapy is needed for papillary carcinoma.

\section{FINAL DIAGNOSIS}

Hyalinising trabecular tumour of thyroid.

\section{REFERENCES}

[1] Karak AK, Sahoo M, Bhatnagar D. Hyalinizing trabecular adenoma-a case report with FNAC histologic, MIB-1 proliferative index and 
immunohistochemical findings. Indian J Pathol Microbiol 1998;41(4):479-84.

[2] Gupta S, Modi S, Gupta V, et al. Hyalinizing trabecular tumor of the thyroid gland. J Cytol 2010;27(2):63-5.

[3] Caraci P, Fulcheri A, Ondolo C, et al. Hyalinizing trabecular tumor of the thyroid: a case report. Head and Neck Pathol 2011;5(4):423-7.

[4] Miller TR, Bottles K, Holly EA, et al. A step-wise regression analysis of papillary carcinoma of the thyroid. Acta Cytol 1986;30(3):285-93.

[5] Santosh KV, Raychaudhuri S, Subramanya H, et al. Cytology of hyalinising trabecular adenoma-like variant of medullary thyroid carcinoma. J Can Res Ther 2011;7(2):189-91.

[6] DeLellis RA, Lloyd RV, Heitz PU, et al. Hyalinizing trabecular tumor. In: World Health Organization classification of tumors. Pathology and Genetics of Tumours of Endocrine Organs. IARC Press: Lyon 2004:104-5.
[7] Rothenberg HJ, Goellner JR, Carney JA. Prevalence and incidence of cytoplasmic yellow bodies in thyroid neoplasms. Arch Pathol Lab Med 2003;127(6):715-7.

[8] Baloch ZW, Puttaswamy K, Brose M, et al. Lack of BRAF mutations in hyalinizing trabecular neoplasm. CytoJournal 2006;3:17.

[9] Casey MB, Sebo TJ, Carney JA. Hyalinizing trabecular adenoma of the thyroid gland identification through MIB-1 staining of fine-needle aspiration biopsy smears. Am J Clin Pathol 2004;122(4):506-10.

[10] Boerner SL, Asa SL. Hyalinizing trabecular tumor of the thyroid gland: much ado about nothing? Am J Clin Pathol 2004;122(4):495-6. 